\title{
Formulating a communication strategy for effective knowledge sharing
}

\section{Yeo Ming Mei, Seow Ting Lee and Suliman Al-Hawamdeh}

School of Library and Information Studies, University of Oklahoma, Schusterman Center, 4502 E 41st St, Tulsa OK 74135-2512, USA

Received 2 February 2003

Revised 16 October 2003

\begin{abstract}
.
Overcoming cultural resistance from a workforce and gaining sufficient buy-in from senior management are both critical to the successful implementation of Knowledge Management (KM) initiatives in any organization. The main purpose of this study is to identify an effective communication strategy at the onset of the implementation process, aimed at helping the workforce to comprehend the need for KM in the organization and to solicit maximum support from the senior management and staff alike. It is based on a case study of the KM initiatives in Singapore's Civil Service College, a training school for public officials. The strategy will also facilitate effective communication between staff so that effective knowledge sharing can take place and provide proactive and reactive communication, to achieve acceptance of and commitment to $\mathrm{KM}$ in the organization. The communication strategy was formulated using inputs collected from focus group discussions and observational field work.
\end{abstract}

Keywords: knowledge management; implementation; communications strategies; organizational culture;

Correspondence to: Professor Suliman Al-Hawamdeh. E-mail: suliman@ou.edu interorganizational communication; organizational acceptance; organizational commitment; knowledge sharing

\section{Introduction}

The strategic role of knowledge in the corporate environment has sparked off intense interest in the concept of using knowledge sharing processes to capture and store organizational knowledge. Moreover, with the rapid advancement of information technology (IT), networking tools have created a potential infrastructure for knowledge sharing and knowledge management (KM) opportunities. As a result, many organizations focus on technology as the solution for their problems, and invest heavily in technical infrastructure to contain or represent knowledge itself. In doing so, they neglect or underestimate the importance of organizational and cultural issues. These play a major role in determining the overall success of KM initiatives. Indeed KM, as with all other change practices, e.g. business re-engineering, is essentially about 'change management.'

Many studies have recognized corporate culture as an essential factor affecting the success of knowledge management efforts [1]. Certainly, organizational culture is often seen as the key barrier to change within an organization. Anderson and Anderson [2, p.26] suggested that when a change in the organization is significant, and requires a new way of being, working, or relating, in order to operate the new environment, leaders are required to change cultural norms for the change to succeed. As a result, there is a need to transform the mindsets of the employees; or else they 
would continue to operate in their old ways, thus stifling the organization's ability to implement the change.

Ultimately, change boils down to people because it is people that make things happen and it is those within the organization that will be executing the changes. However, change will occur only if a change initiative is properly communicated by management. As pointed out by Allee [3, p.214]. 'Without addressing the underlying beliefs and assumptions people hold about knowledge, learning and sharing,' the results of implementing KM initiatives often disappoint.

\section{Communication and resistance to $\mathrm{KM}$ change}

Change efforts that stumble often mismatch their speed and drive with the degree of change required, often moving far too quickly and involving too few people. According to Connor [4], crucial success factors for change management are recognized as: (1) commitment from senior management; (2) nature and intensity of resistance to change; (3) culture of the organization; and (4) knowledge and skill of the change agents, those who help to execute the change.

Studies have shown that nearly two-thirds of major changes are unsuccessful and, according to Fortune 500 executives, the primary reason for failure is not a lack of skill or resources, but resistance to change [5]. Klein [6] suggested that the key reason organizational changes often flounder is because not enough strategic thought is given in communicating the rationale, the progress and the impact of the change. He believed that communication is as important as the changes that are planned and carried forth. Many of the difficulties that are associated with significant change can be more easily dealt with if there is strategic thinking about what and how to communicate. Truly, the transformation of organizational culture is impossible unless many people are willing to participate and help, often to the point of making short-term sacrifices. Yet, employees will not make sacrifices, even if they are unhappy with the status quo, unless they believe that useful change is possible. Thus, Kotter [7, p.11] argued that "without credible communication, and a lot of it, the hearts and minds of the troops are never captured'.

Organizations are increasingly aware that knowledge cannot be treated as an organizational asset without the active and voluntary participation of the communities that are its true owners. As a result, there is a need to rethink reward structures, organizational forms, and management attitudes towards the notion of employees as volunteers of knowledge. However, the question of how best to convince the staff to be volunteers of knowledge remains debatable. A major problem that faces all organizations is the issue of how to persuade, coerce, direct or otherwise get people within organizations to share their information [8]. Some companies prefer to coerce employees to share their knowledge through a series of rigid policies. Unfortunately, employees today are no longer trusting, naive or have an unquestioning deference to authority [9]. They may have also formed their own values and perceptions, and therefore be more resistant to any change that they view as not beneficial to their welfare. Ash [10] maintained that effective communication is essential to the success of any KM program. He believed that effective communication is vital in a decentralised, flattened organizational structure that can no longer depend on command and control from the top down to achieve corporate goals and objectives. Similarly, an article by the American Productivity \& Quality Centre (APQC) 11 argued that investing time in communicating $\mathrm{KM}$ benefits to the staff, simplifying the decision and soliciting feedback could alleviate problems such as a lack of participation and interest in $\mathrm{KM}$ initiatives within the organization.

A large body of change literature supports the use of communication to counter any resistance to change. Oliver [9, p.103] explained that firms that cannot control their employees' hearts can at least attempt to influence their souls through internal lobbying, mission statements and occasionally codes of ethics and value statements. He maintained that it is essential to communicate upwards as well as downwards in order to trigger positive employee involvement so as to harness expertise. Furthermore, it is also necessary to select communications media that support not only the preferred vehicle but also genuine partnership [9, p.19]. According to Quirke [12, p.133], although communication may not change people directly, it would at least remove barriers to change.

Almost every organizational change creates some form of reluctance or resistance, and resistance to change is a major problem that can develop at any point in the change process [12] [13]. However, many authors suggest that resistance can play a useful role in organizational change [5] [14]. The key to successful change management lies in understanding the potential effects of a change initiative on these stakeholders. It is important to anticipate and understand the possible reactions that employees may have towards proposed changes. With this understanding in place, it 
is easier to manage and resolve these negative reactions and to ensure that they will not hamper the implementation process. Galpin [14] argued that understanding the reasons for resistance and working with it rather than against it will aid greatly in creating a smoother process of change. The lack of understanding often results at best in frustration and at worst in dysfunctional behavior, i.e. employees acting out against the change, the initiators of change, and the organization itself [14, p.43]. Understanding why people resist change could be useful in developing a well-thought-out communication plan.

A common complaint from management is that most staff members simply do not appreciate the complexity and the difficulty of the environment in which the organization operates [12]. Thus, it will be very difficult to persuade these employees to change when they do not understand how change, for example a KM initiative, can improve their organisational competitiveness in a volatile environment. Therefore, it is essential that employees are educated about the benefits of the change, such as the need to take advantage of KM to improve an organisation's competitiveness in the global economy, and how it benefits each employee. To begin with, such information should be based upon the concerns and questions of the management and employees. Addressing their concerns and answering their questions at each stage of a change process will help them move up to the next level of the process. According to Galpin [14], the second level of change, the ability of the staff, can be addressed through training and education. In order to change, people are likely to need new skills to function in their future roles, such as working in teams, operating new systems, or following revised procedures. Management also requires new skills to create teams and foster teamwork, coach employees in new skills, and apply new procedures. Because ability has a profound impact on the willingness that people have to undertake new activities and make changes, training becomes an integral part of communication and the change process [14, p.44].

Indeed, people's response to change depends largely on their perception of the proposed change and on the effects they think the change will have on their needs and aspirations 15 . Once they experience ambiguity, they may engage in search behavior that may appear to be resistance from the perspective of those initiating change. Ulrich and Wieland [13] believed that ambiguity may not lead to resistance if individuals are permitted a degree of control over their destiny, as in the case when they are encouraged to participate in the change process [13, p.427]. Indeed, participation in the planning phases of change provides not only opportunity for control but also clarity about the nature of the intended change.

The involvement of senior management in the communication process will send signals to the organization about the priority of change. In addition, the more the individual benefits of change are communicated, the more individuals' ego will be addressed and their willingness to change encouraged. Hence, Galpin [14] maintained that the top level of change is actually 'willingness.' Accordingly, willingness or commitment to change can be increased by several specific actions: (1) establishing individual and team performance goals aligned with the intended changes; (2) measuring people against the goals; (3) establishing effective two-way coaching and feedback mechanisms, and (4) rewarding and recognizing people for achieving the goals and implementing the changes [14, p.44].

\section{Choice of communication strategy in a KM context}

The use of strategic communication as part of $\mathrm{KM}$ strategy has not featured heavily in the KM literature. Almost none of the case studies reviewed during the course of this research demonstrated the use of a communication plan to secure buy-in from the employees and management. While there are many generic communication strategies that are applicable for those companies undergoing change, there are hardly any tailored for the use of KM.

This study focuses specifically on designing a communication strategy as an integral part of KM strategy. It is based on a case study of the Singapore Civil College (CSC). CSC is the main learning institution for civil servants in Singapore. Working closely with Singaporean government departments and a network of public and private sector trainers from both Singapore and overseas, CSC offers programs and courses in leadership, policy development and public administration, among many. This study aims to adapt some of the generic communication strategies to develop a prototype communication plan aimed at promoting KM initiatives in CSC and securing buy-in and support from staff and senior management.

The key element in a good communication plan is the ability to rally as much support as possible for the new vision. One of the ways of ensuring support is by creating ownership through staff involvement in the process of $\mathrm{KM}$ implementation. In this context, own- 
ership means that employees think of the plan as their plan, and not simply the management's plan [16]. Employees can be involved in the process of change by providing feedback to the management on the proposed changes. The feedback is next reviewed by management, which then implements some of the better ideas. This allows the staff to have an opportunity for control and also provides clarity about the nature of the intended organizational change.

Another way to create buy-in for the knowledge management initiatives is to build a momentum of enthusiasm through timely communication. The change literature has shown that when change is dictated from the top down, there is anxiety at the lower rungs of the hierarchy. Timely communication with the staff would allay fears and reduce possible resistance to the new initiatives and the imminent change. In fact, Hadley [16] claimed that regular communication would help create enthusiasm from within the organisation. The practice of open, frank, regularly scheduled communication can not only stop rumours from brewing within the company, but also build a momentum of enthusiasm and support.

There are three important components to consider when developing a communication strategy: (1) the stakeholders; (2) the message of change; and (3) the communication vehicles that are used to deliver the message. Quirke [12] proposed an escalator model that can be used to develop a communication strategy, whether for communicating change or for sustaining change. The escalator metaphor conveys the notion that it is a continual dynamic process and the idea is to keep all members of an organization moving up the escalator, from being merely aware of the change to the stage of being committed to the organizational change. Generally, the more the organization needs an employee to move towards the upper end of the escalator, the more face-to-face communication is needed and the more time is involved [12, p.126]. At the bottom of the escalator, the focus is on the distribution of information to a wide and passive audience. The balance then shifts to greater dialogue and face-to-face communication as it moves towards the middle of the escalator. However, the focus will shift to management willingness to listen and thus doing less talking at the top end of the escalator.

\section{Methodology}

There are two parts to this research problem. For the first, we need to understand how organizational communication can be used to reduce cultural negativity toward knowledge management and in doing so, foster support and encourage buy-in from both the management and staff. The second part involves collecting information on the types of communication tools that are used within the organization that would be effective in fostering positive support for the KM implementation. Breaking down the research problem into two parts will help us understand the intricate dynamics involved.

Two methods were used to collect information observational study and focus group. There are three major types of observational study that exist in business research: non-participant observation, participant observation and ethnography. In non-participant observation, the observer is not directly involved in the situation to be observed. Usually, non-participant observation includes naturalistic observation, simulation observation, case studies and content analysis. In the case of this study, it is most feasible to employ the use of non-participant observational study, specifically the case study approach, because it allows an in-depth investigation of an organization. Additionally, observational data can be collected on inanimate objects such as files as well as on human beings 17 .

According to Morgan [18], observational study is superior for studies on roles in organizations because there are three advantages: (1) the ability to collect data on a larger range of behaviours; (2) a greater variety of interactions; and (3) a more open discussion of the research topic [18, pp. 16-17]. Hence, in this study, observational research methodology can be used to observe the types of communication tools that the staff are more partial towards.

The second method, focus group interviews, was conducted to understand how communication can be used to enhance the implementation of KM policies within CSC. The target group for such interviews was those personnel who would be directly promoting KM to the organization, for example, the 'KM champion', the KM department, etc.

The principal value that focus groups have to offer to a research project based on observational study is the concentrated insight into participants' thinking on a topic. Morgan [18] argued that this is especially useful when a researcher is entering a field site that differs sharply from his or her prior experience - in this case, the CSC environment. Focus groups provide initial exposure to the typical experiences and perspectives of those one is about to observe. Thus the focus group interviews were beneficial in allowing the researcher to gain insights into the organization and the difficulties 
of launching KM initiatives, which a mere observer would not understand.

Field or observational research is extremely useful when a researcher is interested in studying a small group of people, for example, staff within a small organization. Such qualitative research methodology is also more flexible and less structured than quantitative research such as surveys or experiments. It allows the researcher to shift direction and follow other leads. Several weaknesses of this approach must be acknowledged. All field researchers face problems of selecting a suitable field site, as well as gaining access to the site, entering the field and developing rapport with members in the field [18]. The ethical dilemmas that typically arise from the use of field research regarding issues of deception and confidentiality are also an issue, as are observer bias, and the difficulty of generalizing the findings [17].

\section{Findings and analysis}

The focus group meeting involved four participants who were CSC staff members directly involved in the proposed implementation of CSC's knowledge management program. The small size of the focus group can be justified on the basis of these participants' direct involvement in the knowledge management initiative. The members of the focus group were helpful and were willing to share and discuss with the researcher on how to further improve the communication plan even after the focus group session was completed. Some key points emerged from the focus group:

\subsection{The need for knowledge management}

One of the focus group participants cited figures from surveys that were conducted in CSC to show that organizational learning in CSC comes primarily from the following sources:

- hard copy documents;

- tacit knowledge from people; and

- experiential knowledge, such as on-the-job training.

The focus group participant felt that it is crucial to tap into these forms of knowledge, so as to enhance the learning of the organization and its staff. Another participant noted that whenever a staff member leaves the organization, there is a loss of information required for subsequent transfer to the new officer, specifically the loss of knowledge about how to execute the job. As a result there is a long learning curve for the new officer to acquire and familiarize himself or herself with the job scope. A KM initiative would have a key role to play in overcoming this.

\subsection{Resistance in the organisation}

The focus group participants agreed that there is always some form and level of resistance to change within any organization and such resistance can actually derail a project or the implementation process if staff reactions and responses are mismanaged. This was a crucial obstacle that had to be overcome before any change initiative could take place in CSC.

\subsection{Communication as a tool for countering resistance}

When asked by the interviewer whether communication could be a useful tool to counter any potential form of resistance within the organization, the participants agreed that it is a viable option. One of the participants commented that a properly executed communication plan is critical in implementing $\mathrm{KM}$ in an organisation for the purpose of countering resistance that may occur during the implementation stage. He maintained that the communication plan should be able to fulfill the maxim of 'the right message through the right communication vehicle for the right people'.

Another interviewee pointed out that the personal communication style of a communicator is also very important, for example tone of voice, body language and mannerisms. Sometimes it is the personal style of the communicator, not the content communicated, that plays a bigger role in the success of a communication plan, the participant explained. The same participant also opined that Asians tend to make statements while Westerners tend to engage the audience. Hence, he felt that people can learn from both types of method when communicating with an audience about the need to change.

\subsection{Objectives of the communication plan}

The members of the focus group produced the following list of objectives that the communication plan should be able to achieve, if executed correctly:

(1) To raise awareness and understanding of the benefits to the target audience of implementing KM initiatives;

(2) To build commitment and ownership by the target audience through communication of project initiatives, goal directions and the successful completion of project milestones; 
(3) To facilitate effective communication between staff so that effective knowledge sharing can take place; and

(4) To provide proactive and reactive communication so as to achieve acceptance and commitment to $\mathrm{KM}$ in the organization.

The focus group reached a consensus that the most important objective of this communication plan is to secure the buy-in from the management and the staff. One of the interviewers felt that the concept of 'buy-in' could be divided into two phases: acceptance of the change, and participation in the implementation process. Another focus group participant, however, proposed that the concept of 'buy-in' should involve four stages. First, the staff should understand the reasons for the need to change. Second, they would accept the need for change. At the third stage, staff would be committed to making the change happen. The final stage involves the learning of new skills to complement the change.

\subsection{Stakeholders}

The focus group identified six target stakeholders whose support is essential in ensuring the successful implementation of KM initiatives in CSC.

- Senior management

- Line managers

- Network leaders

- Human Resource Department

- Finance Department.

The focus group participants felt that CSC's top management's support for the KM program must be visible and present throughout the implementation. They argued that if the leaders' support for the program is not consolidated or if they are viewed as not 'walking the talk,' their subordinates will deem the change not genuine and therefore be likely to withhold their support for the program.

One member of the focus group, however, questioned the necessity of including network leaders as part of the targeted stakeholders. His argument was that so long as there are no existing communities of practice, there will not be any network leaders. However, he was soon convinced of the necessity of network leaders when the interviewer clarified the definition of network leaders, which can also describe those who are leaders of informal networks within the organization. In the context of CSC, those personnel are lower in rank but they possess substantial power, individual influence or charisma. They are considered network leaders because they are able to command people's respect and are backed with a wealth of experience of working in CSC. These informal network leaders would be well equipped to help to disseminate information. Thus, their support is vital in helping to convince the employees of the benefits of KM, building up a network of knowledge sharing and enhancing the flow of knowledge within the organization.

All participants in the focus group concurred that all CSC employees should be targeted in the communication plan. They understood that the need to change the mindsets of the employees is very important, as failure to do so would unravel the efforts to implement change. The breakthrough in the mindsets of leaders and employees is the key to transforming the culture of the organization to suit the change process. Without targeting the mindsets of the employees, the transformation of culture that is required to support the change process will not take place, even if there is support from the top management. Hence, the securing of freely given support from all employees is quintessential to the success of the change process.

The focus group participants felt that the Human Resource (HR) Department and the Finance Department should be targeted as well. The support from these two departments is needed before the implementation of the program is carried out. One of the group members argued that since the HR Department is in charge of HR policies (which affect CSC staff directly), their willingness to endorse certain policies is necessary for $\mathrm{KM}$ initiatives, for instance in formulating incentives and rewards schemes that encourage knowledge sharing within CSC. The HR Department's endorsement is also required if there is a need to tie in knowledge sharing as a component of staff evaluation, or as a part of the performance management yardstick.

Likewise, the endorsement of the KM program by the CSC Finance Department is equally important because the latter is responsible for allocating budgeting resources for the various communication programs, such as setting up communities of practice, seminars and workshops, etc. Moreover, incentive schemes must also be approved and supported by the Finance Department, together with the main budgetary support to finance the KM undertakings in CSC.

\subsection{Format of communication message}

The focus group participants suggested that the components of the key message should be made clear. Therefore the suggested components of the message are as follows: 
- current situation assessment;

- identification of gaps;

- value proposition; and

- steps ahead (including recommended policies, etc.).

The focus group members felt that the renaming of the components would aid the stakeholders to understand the message more clearly.

One of the members also pointed out that under 'value proposition', the savings or benefits should be generic, rather than specific. He argued that in general, people are motivated by different benefits or savings, i.e. not all employees would appreciate monetary saving that is deemed possible by implementing KM. Thus, the benefits should be emphasised as different aspects, such as 'better working environment' or 'savings in time' for CSC employees; and 'savings in monetary terms' for CSC management. Hence, the focus group participant felt that it would be logical to customize the key message according to the target stakeholders.

\subsection{Types of communication vehicle}

Based on observational study, some communication vehicles clearly work better than others in CSC: e-mail, video clips, story telling, training courses and conference presentation. The issue of communication vehicles was also discussed in the focus group. Participants observed that some vehicles may work well in other organizations but not in CSC. These communication vehicles include memos, cascade briefings, and leveraging on existing communities of practice, etc. First, electronic mail or e-mail is considered the most typical means of communication in CSC. Letters and memos are not effective because they are no longer widely used. Cascade briefings are not considered to be useful in CSC because there is always a tendency, according to the focus group, for the CSC middle management to distort or withhold information from the rest of the staff. Moreover, cascade briefings are time consuming and may not be practical in an organization with a tight implementation schedule such as CSC. Finally, it was observed that there are no existing communities of practice within CSC. Hence, it would not be impossible to leverage existing communities of practice. The focus group felt that it would be easier for the organization to develop and leverage new communities for this purpose.

There are clearly pros and cons in using different communication tools. One of the focus group partici- pants felt that conference presentations might not allow as much feedback as desired when compared to smaller group meetings. However, all the participants agreed that conference presentations are more cost-effective in terms of time, and that the method does allow immediate feedback if a feedback session is incorporated into the conference presentation. Another participant commented that although story telling is a good communication tool, there might be some difficulty in finding analogical stories to share with the audience the message to be conveyed. Another member of the group responded by pointing out that stories do not have to be 'true blue' stories, but can be mere analogies that are used to illustrate or emphasise a key point. It is the person who delivers the story that makes the difference in whether the audience understands the underlying message or not. The focus group also felt that story telling should be reinforced with a multimedia presentation so as to bring across the message more effectively.

\section{Formulation of the communication strategy}

Based on the information gathered, a communication strategy was formulated to accomplish the primary aim of reducing cultural negativity towards the implementation of KM in CSC. Specifically, the strategy focuses on securing maximum buy-in or commitment from the management and employees to support the implementation process.

There are three phases: preparation, mobilization, and implementation. These three phases are divided into five smaller stages: (1) support and commitment; (2) awareness; (3) understanding; (4) support; and (5) commitment. These five stages, initially distinct, affecting management and employees differently, would later merge to form a unified path of organizational change.

For management, a communication plan should begin with securing the 'support and commitment' of stakeholders to KM. For employees, the 'awareness' stage relates to being made aware of the objectives of $\mathrm{KM}$ implementation by management. Next, management and employees must be guided through the 'understanding' stage, whereby they are made to comprehend the new policies and processes that are implemented to sustain the smooth running of KM initiatives. Following that, management and employees will reach the 'support' stage, where they participate in the various 
knowledge sharing and training sessions. 'Involvement,' where the staff members are encouraged to be involved in the process happens in all the stages. Lastly, they would attain sufficient commitment to ensure the success of the KM project.

The communication plan attempts to tailor the right message to the right audience through the use of the right communication vehicles. Hence, the communication strategy is based on a particular sequence.

First, the communication plan would have to identify the stakeholders involved in the change process. This is imperative to the success of the communication strategy because these people are usually the ones who would be affected by the changes imposed by the KM implementation, and therefore their cooperation is vital. Stakeholders usually include staff such as senior management, middle management, employees and even external partners such as suppliers and buyers. Once the stakeholders are identified, the organization can then focus on the possible concerns that these people have and provide answers to address their concerns and questions. Such actions would be extremely effective in defusing any possible resistance to change or deflecting any cultural negativity towards change. Typically in any organization, the stakeholders are local line managers, executive leaders and the network leaders. This is because the rest of the staff usually looks to them for leadership. If these stakeholders were not convinced about the change, it would be difficult to induce the rest of the staff to change as well.

Second, the communication plan must identify the key message that is to be sent to each stakeholder to accomplish the objectives. Although APQC [11] recommended that $\mathrm{KM}$ be best linked to business objectives instead of trying to explain to the target audience the abstract theories of KM, it must be noted that different audiences may be concerned about different objectives, for example the staff may be interested in time savings while the senior management may be more interested in financial savings. Hence, this communication plan proposes to have a key message, which can be adapted to suit each stakeholder. This means the key message remains the same throughout the communication plan, the difference being a different emphasis on different objectives when the key message is delivered to different stakeholders.

The proposed structure of the key message consists of three components. The first component deals with current situation assessment and identification of gaps within the workflow processes. The second component proposes value propositions, where the objective is to quantify the results of possible improvements and their impact on the organization. The final component recommends steps for the organization to take, so as to create a sense of security within the target audience by demonstrating through plans and actions that it is possible to achieve the improvement identified and evaluated in the previous stages.

Third, the communication plan should identify the right communication vehicles that will be used to communicate with each stakeholder. This is a critical factor because different stakeholders respond to different communication vehicles. This is also in line with Quirke's [12] suggestion that different communication vehicles can help to effect a certain degree of change depending on the type of staff involvement.

Fourth, the communication strategy should incorporate a timeline for the communication plan.

Finally, the plan should identify the people who will be accountable for developing and delivering the messages.

\subsection{A communication strategy plan for CSC}

In the Communication Strategy Plan for CSC (see Figure 1), preparation refers to the phase whereby the organization is preparing its staff for the change in work process in small increments such as making them aware of a need for change. The mobilization phases come about when there is a need to encourage the staff to be proactive and participate in the change process. The implementation phase is typically the final phase in a communication strategy. At this stage, employees would be fully committed to the change process when KM is implemented in the organization. During the preparation stage, the project team should be able to identify two things: the stakeholders and the key message for the stakeholders. In the case of CSC, the focus group identified six types of stakeholders in the organization: senior management, line leaders, network leaders, the Finance and HR Departments, and employees.

The senior management of CSC would include the Dean and CEO, the Directors and Deputy Directors as well as senior managers. The commitment from top management is required because they would need to visibly and consistently validate $\mathrm{KM}$ policies by 'walking the talk'. As for the line leaders and informal network leaders, they are essential in initiating change because of their leadership qualities, charisma and the respect that they command from their subordinates and peers. The Finance Department and HR Department were also targeted in the proposal because both 


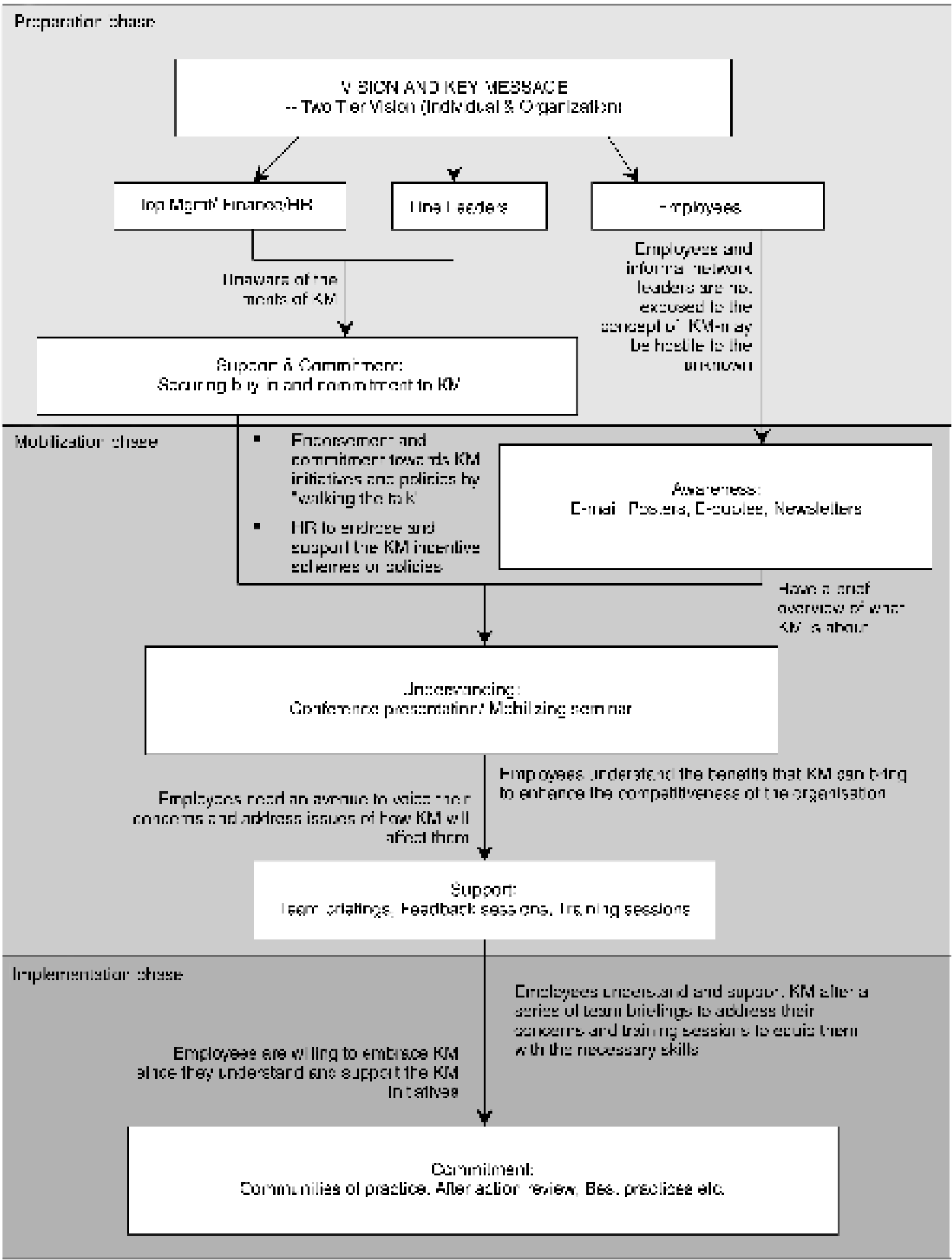

Fig. 1. Communication Strategy Plan for CSC KM Program. 
departments would have to play an active role in ensuring the smooth implementation of the project, as discussed earlier. Finally, CSC employees are also targeted. As Anderson and Anderson [2] had argued, if the mindsets of the employees are not targeted, the transformation of culture that is required to support the change process would not take place at all [2, p.26].

The communication message is made up of two components: the vision and the message. In regard to organizational vision, CSC's KM vision has two tiers. It caters to both the individual and the organization.

The KM vision for the individual is that the latter should have the following attributes: (1) a sense of belonging to CSC; (2) an assurance that CSC recognizes his/her knowledge; and (3) an eagerness to learn and share knowledge.

As for the organisation, the KM vision envisages CSC as an organization that is enterprising, and has a generative learning culture as well as swiftness in delivering innovative value-added customer solutions. In order to achieve this, the $\mathrm{KM}$ mission is therefore to consistently exceed customer expectations and integrate internal expertise through the generation, transfer and application of knowledge as well as the cultivation of a culture of learning through the practice of knowledge sharing within CSC.

Towards the end of the preparation phase, the project team must be able to gain support and commitment from at least four stakeholders: i.e. the top management, the line managers, the Finance Department and lastly the HR Department. These four stakeholders must be committed and fully supportive of the change efforts as they are the key people who provide the leadership and the necessary infrastructure for the implementation of the KM initiatives. In order to secure buy-in from these stakeholders, the communication plan proposes face-to-face meetings with stakeholders to communicate the merits of the project and to persuade them to support the project. Once their support and commitment are secured, then the project can proceed to the second phase: mobilization.

The mobilization phase involves rallying employees towards change in incremental stages. Initially, other stakeholders such as the informal network leaders and employees that were not involved in the preparation stage will be unexposed to the concept of KM and therefore may be hostile to the unknown. According to Galpin [14], people need knowledge. Therefore, the communication strategy aims to slowly expose these people to the concept of KM by giving them a brief overview of KM through email, posters, e-quotes, and newsletters. Once people are aware of KM, the communication plan would seek their understanding by organizing conference presentations to address the issue of change.

As soon as there is some level of understanding about the organizational benefits of implementing $\mathrm{KM}$, the subsequent step would be to solicit staff support by organizing team briefings and feedback sessions to give employees avenues to voice their concerns and feedback to the project team. Training sessions at this stage would enable people to cope with changes effectively. This is in line with Galpin's [14] reasoning that in order to change, people are likely to need new skills in order to function in their future roles, such as operating new systems, or adjusting to new teamwork, etc. Ability, Galpin reasoned, has a profound impact on people's willingness to undertake new activities and make changes. Hence training should become an integral part of communication and the change process [14, p.44]. After the series of steps taken during the mobilization phase, the employees would be more willing to embrace KM since they would now understand and support the KM initiatives when implemented.

\section{Conclusion}

Many KM initiatives have failed because organizations have not taken into account organizational resistance towards change. This study proposes the establishment of a good communication strategy plan at the onset of any KM implementation process to help counter any cultural negativity about change, and to solicit maximum buy-in and support from senior management and staff alike. A good communication strategy must also take into account the reasons for resistance and work with them so as to create a smoother process of change. As such, the key element in a good communication plan is the ability to rally as much support as possible for the change by creating ownership through employee involvement in the implementation process.

The critical objectives for such a plan are raising awareness; increasing understanding of the benefits of implementing $\mathrm{KM}$ initiatives to the target audience; building commitment and ownership by the target audience through communication of project initiatives, goals and directions; facilitating effectual communication between staff so that effective knowledge sharing can take place; and lastly, providing proactive and reactive communication to achieve acceptance and commitment to KM initiatives in the organisation. The insights gained from this study are important steps 
toward formulating an effective communication strategy in KM implementation.

\section{References}

[1] J. Kotter and J. Heskett, Corporate Culture and Performance (Free Press, New York, 1992).

[2] D. Anderson and L. Anderson, Beyond Change Management (Jossey-Bass/Pfeiffer, San Francisco, 2001).

[3] V. Allee, The Knowledge Evolution (Butterworth-Heinemann, Boston, MA, 1997).

[4] D. Connor, Leading at the Edge of Chaos: How to Create the Nimble Organisation. (John Wiley \& Sons, New York, 1998).

[5] D. Waddell and A. Sohal, Resistance: a constructive tool for change management, Management Decision 36(8) (2001) 543-548.

[6] S. Klein, A management communication strategy for change, Journal of Organisational Change Management, 8(2) (1996) 32-46.

[7] J. Kotter, Leading change: why transformation efforts fail, Harvard Business Review on Change, 73 (1995) 120.

[8] B. Gupta, L. Lyer and J. Aronson, Knowledge management: practices and challenges, Industrial Management \& Data Systems 100(1) (2000) 17-21.
[9] S. Oliver, Corporate Communication, (Kogan Page, London, 1997).

[10] J. Ash, Communication Missing from KM's Core Strategies (2000). Available at: www.knowledgepoint.com.au/ knowledgemanagement/Articles/KM_JA001.htm (accessed 16 October 2003).

[11] American Productivity \& Quality Center (APQC), Socializing Knowledge Management (2001). Available at: http://seminare.design.fh-aachen.de/orgadesign/discuss $/ \mathrm{msgReader} \$ 23$ ?mode $=$ day (accessed 16 October 2003).

[12] B. Quirke, Communicating Change (McGraw-Hill, New York, 1995).

[13] R. Ulrich and G. Wieland, Organisation Theory and Design (Richard D. Irwin, London, 1980).

[14] T. Galpin, The Human Side of Change: A Practical Guide to Organisation Redesign (Jossey-Bass, Boston, MA, 1996).

[15] F. Mann and F. Neff, Managing Major Change in Organisations (Foundation of Research on Human Behavior, Ann Arbor, 1961).

[16] K. Hadley, Creating Buy-in for Your Strategic Plan (1997). Available at: www.fed.org/onlinemag/aug97/ hadley.html (accessed 16 October 2003).

[17] L. Gay and P. Diehl, Research Methods for Business and Management (Maxwell Macmilllan International, New York, 1996).

[18] D. Morgan, Focus Groups Qualitative Research Method (Sage Publications, Thousand Oaks, 1988). 